Received: 3 August 2014, Accepted: 3 August 2014

Edited by: G. Martinez Mekler

Licence: Creative Commons Attribution 3.0

DOI: http://dx.doi.org/10.4279/PIP.060004

www.papersinphysics.org

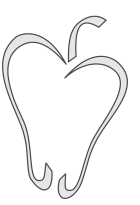

ISSN 1852-4249

\title{
Commentary on "Critical phenomena in the spreading of opinion consensus and disagreement"
}

\author{
Franco Bagnoli ${ }^{1 *}$
}

The authors of Ref. [1] study a variation of the voter model in which the neighbors of a cluster of agents agree or disagree with the nearest member of the group according with the agreement inside the group.

The article is interesting and well written. The authors show that the phase transitions towards the full consensus obey definite scale relations.

I have some points that I think should be considered by the authors. The first one is about Fig 1 in their paper. For $p_{D}=p_{C}=1$, the system presents three absorbing states: full consensus "black", full consensus "white" and maximal disagreement, i.e., staggered "black-white". It seems from the figure that the system reaches the "black" absorbing state at a time about 2300 and 4700 , and the staggered one at time about 7500 . However, in all these cases the presumed absorbing state is abandoned. This is surely due to the fact that what is shown is only a portion of the lattice; the figure is misleading and should be replaced with a picture of the whole lattice, where the "annihilation" of the random walkers corresponding to the boundaries of the clusters could be observed.

The second point is about the correspondence with annihilating random walks. It seems to me that in one dimension for $p_{C}=p_{D}=1$, the dynam-

\footnotetext{
*E-mail:franco.bagnolI@unifi.it

1 Department of Physics and Astronomy and CSDC, University of Florence, via G. Sansone 150019 Sesto Fiorentino, Italy.
}

ics of the model corresponds to that of annihilating random walkers, like in the voter model, except that in this case there are two types of walkers that do not annihilate among themselves. Let me call these two walkers types $\mathrm{L}$ and $\mathrm{R}$. The portion of the lattice between two L corresponds to the pattern $(10)^{*}$ (odd rows black, even-rows white), that between two R is a pattern (01)* (even rows black, odd-rows white), that between one $\mathrm{L}$ and one $\mathrm{R}$ is $0^{*}$ and that between one $\mathrm{R}$ and one $\mathrm{L}$ is $1^{*}$. $\mathrm{R}$ and $\mathrm{L}$ can cross, two $\mathrm{L}$ or two $\mathrm{R}$ annihilate. If this correspondence is true, one should be able to interpret the scaling laws in the context of annihilating random walks. For $p_{C}=p_{D}$ different from 1, one might find some correspondence with branching annihilating random walks and directed percolation.

The final concern is about the generalization of the rule to non-regular lattices. Social networks are surely not regular, and often show a broad distribution of connectivity. How could the rule be generalized to these cases?

[1] A Chacoma, D H Zanette, Critical phenomena in the spreading of opinion consensus and disagreement, Pap. Phys. 6, 060003 (2014). 